\author{
Richard Saage
}

\title{
Toleranz und Demokratie Zur Vorgeschichte und Zukunft des liberalen Pluralismus
}

Dass Toleranz im engen Konnex mit dem Partizipationshandeln der Bürger jenseits staatlicher Reglementierung zu sehen ist und der eine Begriff den anderen bedingt und umgekehrt, ist für das deutsche Publikum alles andere als selbstverständlich. Noch immer kann die von Bodin, Hobbes und Carl Schmitt geprägte Vorstellung große Plausibilität für sich reklamieren, dass erst der starke Staat bürgerliche Freiheiten und Toleranz in dem Maße ermöglicht habe, wie er in der Frühen Neuzeit die pluralen religiösen Kräfte zähmte und neutralisierte. Im Folgenden soll demgegenüber die These vertreten werden, dass dieses Paradigma insofern einseitig ist, als sich gleichursprünglich eine alternative Linie des politischen Denkens durchsetzte, die umgekehrt argumentierte: Danach können sich Toleranz und Bürgerfreiheit erst dann voll entfalten, wenn sie den Staat in den Dienst ihrer eigenen Garantie stellen. Nur unter dieser Bedingung hat der freiheitliche Pluralismus eine Chance, wie Ernst Fraenkel ihn in der Zweiten deutschen Republik nachhaltig propagierte (Fraenkel 1968).

Inhalt

1. Einleitung

2. Toleranz und Bürgerfreiheit im starken Staat

3. Differenzen zwischen dem etatistischen und dem liberalen Toleranzmuster 461

4. Das Scheitern des autoritären Antipluralismus 463

5. Bewährungsproben des liberalen Pluralismus $\quad 465$

6. Die Dialektik von Relativismus und Fundamentalismus 467

Literatur

\section{Einleitung}

Die These dieses Beitrags ist, dass es im Kontext der Entstehung bürgerlicher Gesellschaften zwei Wege zur Etablierung religiöser Toleranz gegeben hat: Das eine Paradigma verweist auf den absolutistischen Staat, der kraft seiner potestas die pluralen Kräfte der religiösen Lager entpolitisiert und dadurch befriedet. Es impliziert freilich das Defizit, Toleranz als Teil der Staatsräson zu betrachten und diese zu ermächtigen, je nach Gesichtspunkten der Machterhaltung religiöse Duldung zu gewähren oder wieder zurückzunehmen. Wie gezeigt werden 
soll, legte dieses Muster die Grundlage des autoritären Antipluralismus, der insbesondere in Deutschland sowohl in der Endphase der Weimarer Republik als auch im Dritten Reich scheiterte. Das andere Paradigma setzte in der Frühen Neuzeit auf den Konsens der pluralen Kräfte der Gesellschaft, die in den Ständeversammlungen (Niederlande) und im Parlament (England) die Toleranz von unten als naturrechtlich begründetes „Geburtsrecht“ institutionell zu verankern suchte: Der Staat wird gleichzeitig in die Pflicht genommen, religiöse Toleranz, die ihm vorgeordnet ist, als individuelles Grundrecht zu schützen. Dieses Modell hat sich bis auf den heutigen Tag als robust und strapazierfähig erwiesen, wie insbesondere an der Entwicklung der liberalen Demokratie in der Bundesrepublik Deutschland abzulesen ist.

Die Einlösung dieses Programms legt folgende Schritte nahe. Idealtypisch vereinfacht, versuche ich zunächst, das Paradigma der Entstehung von Toleranz und bürgerlichen Freiheiten im Schoße des starken Staates am Beispiel der Rekonstruktion dieses Vorganges durch Carl Schmitt zu verdeutlichen (Teil 2). Diesem etatistischen Muster innerstaatlicher Befriedung angesichts der religiösen Bürgerkriege des 16. Jahrhunderts in Frankreich, wie Roman Schnur es an der Toleranzkonzeption der so genannten Politiques, deren Sprecher Jean Bodin war, festmacht, konfrontiere ich dann als Alternative die Legitimationsstrategien religiöser Meinungsfreiheit im Aufstand der Niederlande in der zweiten Hälfte des 16. Jahrhunderts und der großen Englischen Revolution von 1642-1649 (Teil 3). Anschließend stelle ich am historischen Fallbeispiel der Weimarer Republik und des Dritten Reiches das Scheitern des autoritären Antipluralismus dar (Teil 4). Ihm konfrontiere ich dann den zivilgesellschaftlichen Pluralismus, wie Ernst Fraenkel ihn im 20. Jahrhundert als erfolgreiches Ordnungsmodell des 20. Jahrhunderts entwickelte (Teil 5). In einem abschließenden Diskurs erörtere ich die Frage, inwiefern die der liberalen Demokratie zugrunde liegende Pluralismuskonzeption geeignet erscheint, insbesondere den absehbaren sozio-kulturellen Integrationsproblemen des 21. Jahrhunderts gewachsen zu sein (Teil 6).

\section{Toleranz und Bürgerfreiheit im starken Staat}

Eine folgenreiche Aktualisierung der etatistischen Rückbindung von Toleranz und Bürgerfreiheit hat Carl Schmitt in seiner Schrift „Der Leviathan in der Staatslehre des Thomas Hobbes. Sinn und Fehlschlag eines politischen Symbols“ (1938) vorgenommen. Er wies den Einwand John Lockes gegen den Hobbes'schen Leviathan zurück, es sei unsinnig zu unterstellen, die Einzelnen würden aus Angst vor Hunden und Katzen es vorziehen, von einem Löwen gefressen zu werden. Hobbes Ziel sei es vielmehr gewesen, „durch den Staat die Anarchie des feudalen, ständischen oder kirchlichen Widerstandsrechts und den darauf fortwährend neu entbrennenden Bürgerkrieg zu überwinden und dem mittelalterlichen Pluralismus, den Herrschaftsansprüchen der Kirchen und anderer, indirekter' Gewalten die rationale Einheit einer eindeutigen, eines wirksamen Schutzes fähigen Macht und eines berechenbar funktionierenden Legalitätssystems entgegenzusetzen“" (Schmitt 1938: 113). Zu einer solchen rationalen staatlichen Macht gehöre die volle politische Gefahrenübernahme und in diesem Sinne die Verantwortung für den Schutz der Staatsunterworfenen. Sei dieser Schutz nicht mehr garantiert, löse sich der Staat auf und die Gehorsampflicht der Untertanen entfalle. Seine „natürlichen Rechte“ fielen dann an die Einzelnen zurück. Solange aber der Staat die 
pluralen Mächte mit ihrem Absolutheitsanspruch neutralisiere und in ihre Schranken weise, schaffe er erst die Bedingung für ein Minimum an öffentlicher Sicherheit, ohne die weder religiöse Toleranz noch Bürgerfreiheiten existieren könnten. Doch deren Grundlage sei die „Relation von Schutz und Gehorsam“ als „Angelpunkt“ der Staatskonstruktion, die sich „,mit den Begriffen und Idealen des bürgerlichen Rechtsstaates sehr gut vereinbaren“ (Schmitt 1938: 114) lasse.

Carl Schmitt selbst aktualisierte den religiösen Pluralismus der auf freiwilliger Grundlage in christlichen Gemeinden vergemeinschafteten Individuen der Frühen Neuzeit, wenn er „die alten Gegner, die ,indirekten“ Gewalten von Kirche und Interessenorganisationen“ im 20. Jahrhundert ,in moderner Gestalt als politische Parteien, Gewerkschaften, soziale Verbände wiedererscheinen“ (1938:116) lässt. Die „Mächte der Gesellschaft“ hätten sich ,auf dem Weg über das Parlament der Gesetzgebung und des Gesetzesstaates bemächtigt" (Schmitt 1938: 116). Dass sie den Leviathan vor ihren Karren zu spannen vermochten, so seine These, erleichterte ihnen ein Verfassungssystem, „dessen Grundschema ein Katalog individueller Freiheitsrechte war. Die dadurch garantierte, angeblich freie Privatsphäre wurde dem Staat entzogen, um den ,freien', d. h. unkontrollierten und unsichtbaren Mächten der ,Gesellschaft‘ ausgeliefert zu werden“" (Schmitt 1938: 117).

Die Konsequenzen dieses Vorganges sind von Carl Schmitt und seiner Schule, deren intellektuelles Spektrum von Ernst Forsthoff und Werner Weber bis hin zu Roman Schnur, Bernard Willms und Reinhart Koselleck reichte, immer wieder variantenreich vorgetragen worden. Um Kirchen und Gewerkschaften herum hätten es die heterogenen organisierten Interessen geschafft, ein Parteiensystem zu etablieren, das die selbst noch im Kaiserreich gültige Unterscheidung zwischen Staat und Gesellschaft durch den Pluralismus der ,,indirekten Gewalten“ mühelos zu unterlaufen vermochte. Es gehöre zu deren Wesen, „die eindeutige Übereinstimmung von staatlichem Befehl und politischer Gefahr, von Macht und Verantwortung, Schutz und Gehorsam“ (Schmitt 1938: 117) zu unterlaufen. Die Konsequenzen dieser indirekten Methode lägen auf der Hand: Parteien und Verbände agierten als private Organisationen, gleichzeitig nutzten sie aber jenseits aller Verantwortlichkeit ,alle Vorteile der Staatlichkeit für sich" (Schmitt 1938: 118) aus. Unter den Bedingungen krisenhafter ökonomischer, sozialer und politischer Rahmenbedingungen steuere ein solcher Pluralismus auf den Bürgerkrieg zu, wie Schmitt in seiner Schrift „Der Hüter der Verfassung“ (Schmitt 1985) in der Endphase der Weimarer Republik nachweisen zu können glaubte.

Da Schmitt ebenso wenig wie Hobbes den Boden der bürgerlich-kapitalistischen Eigentümergesellschaft verlässt, kann ihnen zufolge der angestrebten Homogenität des Staates keineswegs eine sozial homogenisierte Gesellschaft gegenübertreten, in der die Individualinteressen vollständig vom mächtigen Leviathan konsumiert werden. Aber deren Artikulation und Durchsetzung im Schutze des starken Staates hat seiner Aufsicht zu unterliegen (Hobbes 1984: 173-184; zusammenfassend Euchner 1987: 67). Es ist nicht schwer, auf dieser Hobbes'schen Folie das antipluralistische Modell Schmitts zu dechiffrieren, das er seiner etatistischen Pluralismuskritik konfrontiert. Strikt lehnte er es wie dieser ab, dass der Staat lediglich als neutraler Schlichter der konfligierenden organisierten Interessen im Namen einer übergeordneten Vernunft agiert, wie es John St. Mill vorschwebte. Vielmehr soll er ganz im Sinne von Hobbes kraft seiner souveränen Entscheidung den sozialen Frieden in der 
Ausnahmesituation herstellen können, ohne den konsensualen Ausgleich zwischen den konkurrierenden Interessengruppen und Parteien abzuwarten.

Zwei Differenzen, die ihn von Hobbes trennen, dürfen nicht verschwiegen werden. Aber man sollte sie auch nicht überbewerten, sind sie doch den verschiedenen Entwicklungsstadien der bürgerlichen Gesellschaft im 16. und 20. Jahrhundert geschuldet. Kannte Hobbes vorwiegend Individuen, so glaubte Schmitt, dass man in industriellen Massengesellschaften zur kollektiven Einheitsstiftung um legitimatorische Größen wie die des „,Volkes“ oder der „Nation" nicht herumkommt. Zum andern konnte ihm zufolge der Staat der Aufgabe der Konfliktbewältigung nur dann genügen, wenn er - im Unterschied zu Hobbes und Bodin - dem demokratischen Zeitalter zumindest ansatzweise legitimatorisch Rechnung trug.

Dieses Ziel strebte Schmitt durch den Rückgriff auf eine Identitätskonstruktion an, deren demokratische Substanz freilich fragwürdig genug erscheint: Sie ist für den Leviathan im Volk selbst verankert, das mit diesem insofern eine homogene Einheit bilden soll, als es angeblich nicht durch organisierte Interessengruppen parzelliert und gerade dadurch befähigt ist, eine Korrektur und Schranke des modernen pluralistischen Parteienstaates darzustellen. Indem das Volk den Leviathan also direkt durch Akklamation „,bestätigt“, bekennt es sich zu sich selbst und rechtfertigt damit den ehernen Rahmen, innerhalb dessen sich eine vom Staat lizenzierte und damit von ihm mediatisierte Interessenvertretung abspielen kann.

Folgt man den Pluralismus- und Toleranzvorstellungen der etatistischen Konservativen und ihrem Konzept der innerstaatlichen Friedensstiftung, so gewinnt man den Eindruck, als verdanke sich der freiheitliche Pluralismus immanenten Defiziten des autoritären staatlichen Integrationsmusters selbst und nicht einer eigenständigen Genese jenseits etatistischer Überformung. So liegt nach Carl Schmitt das Strukturdefizit des Hobbes'schen Leviathan in dessen Unterscheidung zwischen ,,privat und öffentlich, Glaube und Bekenntnis, fides und confessio, faith und confession" (Schmitt 1938: 85). Schon wenige Jahre nach dem Erscheinen des Hobbes'schen Hauptwerkes sei der Blick des ,ersten liberalen Juden“, nämlich Spinozas, auf die einschlägige Textstelle gefallen. „Er erkannte in ihr sofort die große Einbruchstelle des modernen Liberalismus, von der aus das ganze, von Hobbes aufgestellte Verhältnis von Äußerlich und Innerlich, Öffentlich und Privat, in sein Gegenteil verkehrt werden konnte“" (Schmitt 1938: 86). Sollte bei Hobbes die Gewissensfreiheit ins Private abgedrängt und damit politisch neutralisiert werden, so kehrte sich jetzt dank Spinozas Intervention dieses Verhältnis um: „Jetzt wird umgekehrt die individuelle Gedankenfreiheit der formgebende Grundsatz und die Notwendigkeiten des öffentlichen Friedens sowie das Recht der souveränen Staatsgewalt verwandeln sich in bloße Vorbehalte. Eine kleine, umschaltende Gedankenbewegung aus der jüdischen Existenz heraus, und in einfacher Folgerichtigkeit hat sich im Laufe von wenigen Jahren die entscheidende Wendung im Schicksal des Leviathan vollzogen“" (Schmitt 1938: 88 f.).

Doch ist diese personalisierte Rekonstruktion der Genesis der liberalen Pluralismustheorie, die Schmitt 1938 in eindeutig antisemitischer Absicht dem angeblich ,zersetzenden“ Geist des Judentums, verkörpert von Baruch Spinoza, Moses Mendelssohn und Friedrich Julius Stahl, zuordnet, leicht als das zu durchschauen, was sie von Anfang an war: ein Akt offenkundiger Geschichtspolitik, von der nur diejenigen geblendet werden können, die im Schatten der ausschließlich etatistischen Sicht der Beendigung der religiösen Bürgerkriege 
in der Frühen Neuzeit verharren. Lange vor Spinozas Hobbes-Rezeption hatte sich seit dem Abfall der Niederlande von der spanischen Krone im letzten Viertel des 16. Jahrhunderts und in der großen Englischen Revolution von 1642 bis 1649 ein alternatives Paradigma der innerstaatlichen Friedensstiftung entwickelt, das institutionell, soziologisch und theoriegeschichtlich eigene Wege zum religiöse Toleranz ermöglichenden innerstaatlichen Frieden beschritt (vgl. zusammenfassend Saage 1981). Um den spezifischen Kern jener Entwicklung zu mehr Bürgerfreiheit als einer „Aufgabe der gesellschaftlichen Kräfte selbst“ (Euchner 1981: 10) am niederländischen und englischen Beispiel verdeutlichen zu können, erscheint eine Komparatistik zwischen diesem Lehrstück und der französischen Entwicklung hilfreich zu sein, die Roman Schnur (1962) zu rekonstruieren versucht hat. Dass der folgende Vergleich keine Vollständigkeit anstrebt, sondern sich ausschließlich von einem typologischen und analytischen Erkenntnisinteresse leiten lässt, sei an dieser Stelle ausdrücklich betont. Worin bestehen also in einer solchen Perspektive die wichtigsten Differenzen auf den oben erwähnten drei Ebenen?

\section{Differenzen zwischen dem etatistischen und dem liberalen Toleranzmuster}

Zunächst ist festzustellen, dass das institutionelle Medium, innerhalb dessen der Bürgerkrieg beendet und Toleranz ermöglicht werden sollte, im französischen und im niederländisch/ englischen Entwicklungsmodell der Frühen Neuzeit nicht unterschiedlicher sein konnte. Schnur weist nach, dass die so genannten Politiques, eine Gruppe staatstreuer Juristen, wie ihr Sprecher Jean Bodin auf die unteilbare Souveränität des absolutistischen Königtums setzten, das mit den Mitteln der Polizei, eines stehenden Heeres und einer funktionierenden Bürokratie die politisierten religiösen Lager „,neutralisiert“ und dadurch die Voraussetzung für eine vom Staat gewährte Toleranz schafft (Schnur 1962). Demgegenüber strebten die oppositionellen Kräfte in den Niederlanden und in England eine Befriedung der Gesellschaft von unten an. Dieses Ziel suchten sie nicht über den starken absolutistischen Staat, sondern zunächst über die Ständeversammlungen (Niederlande) und später über das Parlament (England) zu erreichen. Beide Institutionen verstanden sich als Repräsentationsorgane des „Volkes“, wenn auch in unterschiedlicher Ausprägung. In den Niederlanden galt das Volk noch als ständisch gegliederte Größe, in England erfolgte der Durchbruch zum modernen Verständnis der Volkssouveränität, die aus dem autonomen Willen mündiger Bürger resultiert. In beiden Fällen handelte es sich jedoch um eine innerstaatliche Friedenstiftung, die tendenziell auf dem Konsens der Bürger, nicht auf der potestas des Staates gründete. In gewisser Weise kehrte dieses Paradigma das Verhältnis von Staat und Toleranz um: Der absolutistische Staat fungierte nicht als conditio sine qua non der Toleranz und der Gewissensfreiheit; er war vielmehr der die Toleranz bedrohende Instanz, die erst niedergerungen werden musste, bevor das demokratische Fundament religiöser und gesellschaftlicher Pluralismen gelegt werden konnte (Saage 1981).

Auch soziologisch unterscheiden sich beide Modelle gravierend. Wie wir sahen, reduzierte Carl Schmitt die etatistische Lösung der Bürgerkriegsproblematik auf die Konzeption der Klassiker des politischen Denkens wie die des Thomas Hobbes (Schmitt 1938). Sein Schüler Roman Schnur versuchte am französischen Beispiel, diese enge Personalisierung 
dadurch zu überwinden, dass er nach einer soziologischen Unterfütterung der etatistischen Toleranzvariante suchte: Er fand sie bekanntlich im Frankreich des 16. Jahrhunderts in den bereits erwähnten Politiques, die zwischen den sich befehdenden Lagern der Calvinisten und der Katholiken eine „dritte Kraft“ darstellten. Zwar wird man von einer einflussreichen juristischen Berufsgruppe reden können, die in den als Berufungsinstanz in Lehensstreitigkeiten zuständigen Parlamenten, vor allem aber in dem die königlichen Erlasse registrierenden obersten Gerichtshof in Paris, ihre Machtbasis hatte. Aber gemessen an soziologischen Kriterien, ist sie kaum als eigenständige soziale Schicht zu klassifizieren (Schnur 1962: 26-40). Auch deutet nichts darauf hin, dass sie mit ihren absolutistischen Bestrebungen eine Überwindung der feudalen Gesellschaftsstruktur Frankreichs angestrebt hätte.

Dagegen waren sowohl die Ständeversammlungen in den Niederlanden wie das Parlament in England Repräsentationskörperschaften des aufstrebenden Besitzbürgertums als einer gesellschaftlichen Klasse, das im Verlauf der Zuspitzung des Kampfes gegen die absolutistischen Kräfte in beiden Ländern partielle Bündnissse mit den Unterschichten einging. Dabei ist von entscheidender Bedeutung, dass sich im Unterschied zu den Politiques in Frankreich die Friedensbemühungen von unten nicht ausschließlich im Rahmen der vorgegebenen Ständegesellschaft abspielten. Zwar sind in der Pamphletistik des Aufstandes der Niederlande keine Hinweise auf ein systematisches antifeudales Programm zu erkennen. Aber faktisch war die Macht der holländischen Handelsbourgeoisie in der Oppositionsbewegung wirksam, für die die feudalen Restriktionen längst zu einer Fessel ihrer frühkapitalistischen Wirtschaftsaktivitäten geworden war. Und in England schlug der antifeudale Impetus auch auf der programmatischen Ebene voll durch, wie die wirtschaftlichen Forderungen der im Parlament tonangebenden Gentry insgesamt, aber auch der Levellers und Diggers in ihren Manifesten unübersehbar zeigen (Saage 1981: 254-258).

Doch wie wirkten sich diese Prozesse theoriegeschichtlich aus? Vereinfacht ausgedrückt, haben wir es in den Niederlanden und in England mit einer Theorieentwicklung zu tun, die sich als klare Alternative zum Machtstaatsdiskurs der Politiques in Frankreich verstand. Für die Vertreter des absolutistischen Königtums waren der Ausgangspunkt ihrer Überlegungen offensichtlich die Gräuel des Bürgerkrieges und das für sie unakzeptable Szenario des beginnenden Zerfalls der französischen Monarchie in autonome Regionen, die sich zunehmend der Zentralmacht entzogen. Selber zum größten Teil Katholiken, war für sie Toleranz eine Frage der Staatsräson und als solche ein Mittel, den Bürgerkrieg zu beenden. Es ging ihnen um eine Art Schadensbegrenzung im Interesse des Staates, nachdem klar war, dass sich der Calvinismus wie ein Flächenbrand über ganz Frankreich verbreitet hatte. Die von ihnen angestrebte Toleranz verblieb in der Disposition des absoluten Monarchen: Er konnte je nach dem Interesse der Machterhaltung und -erweiterung des Staates - Toleranz gewähren oder aber einschränken (Forst 2003: 181-190).

Was demgegenüber in der Pamphletistik des Aufstandes der Niederlande gegen die spanische Zentralgewalt bereits angelegt war, kam im Verlauf der großen Englischen Revolution zum vollen Durchbruch. Zwar legitimierte sich die Opposition in der Anfangsphase des Konflikts noch immer durch den Rekurs auf den - allerdings jetzt schon individualistisch interpretierten - Feudalvertrag der Magna Charta. Doch in dem Maße, in dem man Kompetenzen der Prärogative an sich riss und ein eigenes Heer aufstellte, war der legitimierende 
Rückgriff auf historische Präzedenzien verbaut: Das parlamentarische Lager ging dazu über, den Widerstand gegen den König mit Kategorien des ahistorischen subjektiven Naturrechts zu rechtfertigen. Der König war nichts weiter als Angestellter des Volkes, das sich über einen Vertrag ursprünglich Gleicher und Freier konstituiert hatte. Da er sich weigerte, die ihm zugeordneten Aufgaben - z. B. den Schutz der Gewissensfreiheit - zu erfüllen, war er abzusetzen, notfalls durch die Gewalt der Waffen (Saage 1981: 115-162).

Die Folgen für die Begründung religiöser Toleranz waren nachhaltig. Weit davon entfernt, ein Instrument der Staatsräson zur Niederhaltung fanatischer religiöser Gruppierungen zu sein, die das Politikmonopol des Staates bedrohten, hatte sich die Legitimationsproblematik grundlegend geändert. Die Ausübung der Religions- und Meinungsfreiheit stieg jetzt zu einem „birth right“, zu einem Grundrecht auf, das der menschlichen Natur immanent und dadurch unveräußerlich war (Forst 2003: 233-245). Es hatte nicht länger mehr den Status eines Geschenks des Staates, das an seine Machtkompetenz gebunden blieb. Als unveräußerliches Menschen- und Bürgerrecht dem Staat vorgeordnet, bestand dessen Aufgabe darin, dieses Recht zu schützen, aber nicht, es zu verleihen oder, wenn dies machtpolitisch opportun erscheint, wieder zu entziehen. Diese neue Form der Begründung religiöser Toleranz eröffnete eine innovatorische Dimension der Bürgerpartizipation, die sich der altständischen Welt Europas nicht mehr assimilieren ließ. Wenn der autonome Wille ursprünglich Gleicher und Freier nicht nur der Ursprung der Toleranz, sondern auch der politischen Ämter war, dann stand nun auf der politischen Tagesordnung, was einst die Polisbürger der Attischen Demokratie als selbstverständlich unterstellten: dass sich die Amtsträger durch Wahl der das Volk konstituierenden Vollbürger individuell zu legitimieren hatten. Dieses Postulat zielte darauf ab, die Amtsträger zu einer Politik zu zwingen, als ob der repräsentierte Bürger selbst handelte.

So gesehen, war von Anfang an ein zwingender Strukturzusammenhang zwischen Toleranz und Demokratie gegeben: Erst unter dieser Bedingung konnte in vertikaler Hinsicht eine angstfreie Kommunikation über das bonum commune und auf horizontaler Ebene der Austausch auch konfligierender Argumente gelingen, ohne den Boden gemeinsamer Wertüberzeugungen zu verlassen. Doch ebenso wichtig erscheint, dass die beiden skizzierten alternativen Wege zur religiösen Toleranz in der Frühen Neuzeit die Grundlagen legten für die antipluralistischen und pluralistischen Strukturen der modernen bürgerlichen Gesellschaften des Westens. Welche Formen nahmen sie an und inwiefern sind sie den Herausforderungen des 20. Jahrhunderts gewachsen?

\section{Das Scheitern des autoritären Antipluralismus}

Die Umrisse des antipluralistischen Modells sind bereits skizziert worden: Partikulare Interessenvertretung nur nach vorheriger etatistischer Lizenzierung; Gewährung von Toleranz nach Maßgabe der Erfordernisse der Staatsräson; Wiederherstellung der strikten Unterscheidung zwischen dem homogenen Staat und der heterogenen Gesellschaft; autoritäre Durchgreifmöglichkeiten der etatistischen potestas, ohne durch Grundrechte der Individuen oder parlamentarische Kontrollen gehemmt zu werden; eine plebiszitär legitimierte Staatsgewalt als Ausfluss eines angeblich homogenen Volkes, der alle zur Durchsetzung ihres souveränen 
Anspruchs notwendigen Zwangsmittel zur Verfügung stehen - das sind die Stichwörter, mit denen dieser antipluralistische Ansatz charakterisiert werden kann.

In Deutschland hatte dieses Modell seinen historischen Test am Ende der Weimarer Republik und dann im Dritten Reich zu bestehen. Beide Fallbeispiele zeigen, dass der etatistische Antipluralismus den Herausforderungen einer in die Krise geratenen bürgerlichen Gesellschaft des 20. Jahrhunderts nicht gewachsen war. Die Diktatur des Reichspräsidenten auf der Grundlage des Art. 48 WRV, gestützt auf Beamtenschaft und Heer sowie legimiert durch ein pseudodemokratisches Plebiszit, für die sich Schmitt zunächst in der Weimarer Republik dezidiert einsetzte, erwies sich als unfähig, das sich eskalierende Zusammenspiel der sozialen, politischen und ökonomischen Krise ab 1929 in den Griff zu bekommen. Die Gründe sind komplex und können hier nicht in allen Einzelheiten diskutiert werden. Doch fallen zwei Aspekte ins Auge, die entscheidend dazu beitrugen, dass diese Konstruktion in der politischen Wirklichkeit nicht auf Dauer zu stellen war: Ihr fehlte eine Massenbasis und eine Elite, die wenigstens über die politische Weitsicht der französischen Politiques im 16. Jahrhundert sowie über ein Konzept der Massenintegration verfügt hätte. Eine Figur wie der General von Hammerstein-Equord, der vor den Gefahren des faschistischen Dezisionismus und seiner selbstdestruktiven Dynamik warnte, blieb im eigenen Lager ungehört und wurde als Randfigur marginalisiert.

Der zweite Test des antipluralistischen Modells, auf den nun die etatistischen Konservativen wie Carl Schmitt und Ernst Forsthoff (Forsthoff 1933) ihre Hoffnungen projizierten, war das Dritte Reich. Hatte Hitler nicht den Parteienstaat, den Verbandspluralismus und das parlamentarische System abgeschafft? War es ihm nicht gelungen, mit der gewaltsamen Liquidierung der sozialdemokratischen und kommunistischen Organisationen der Arbeiterbewegung und der mit der Reichswehr konkurrierenden Führungsschicht der SA die Gefahr des Bürgerkriegs zu bannen? Und verfügte das nationalsozialistische Regime nicht über das, was einer auf Dauer gestellten Diktatur des Reichspräsidenten fehlte: eine veritable Massenbasis? Doch die mit diesen Fragen verbundenen Hoffnungen auf einen homogenen Staat sollten sich bald als Chimäre erweisen. Was Mussolini in Italien zumindest ansatzweise zu gelingen schien, wurde von Hitler in Deutschland von Anfang an unterbunden: die vollständige Etatisierung der faschistischen Bewegung (Saage 1987: 121-199). Zwar ließen die Nazis die staatliche Struktur der deutschen Polizei scheinbar unangetastet. Aber ihr Chef, Heinrich Himmler, war zugleich der Garant ihrer Bindung an die Interessenlage der „Bewegung“ und ihres Loyalitätsverhältnisses zum charismatischen Führer (Broszat 1986: 326-336). Neben den Mischmodellen aus staatlichen und faschistischen Elementen war darüber hinaus das Dritte Reich durch ein ganzes Netz nationalsozialistischer Sondergewalten charakterisiert, die am traditionellen (preußischen) Staat vorbei auf der Basis von Führerbefehlen agierten und dabei, untereinander im regellosen Sinne ,pluralistisch“ um Einflusschancen konkurrierend (Bast 1999), der staatlichen potestas systematisch Machtanteile entzogen.

Was Schmitt 1938 allgemein konstatieren zu müssen glaubte, ließe sich auch auf den Staat des Dritten Reiches anwenden: „Alle untereinander so feindlichen indirekten Gewalten waren sich plötzlich einig und verbündeten sich zum ,Fang des großen Wals‘. Sie haben ihn erlegt und ausgeweidet" (Schmitt 1938: 124). Mit großem Recht hat Franz Neumann in seiner Studie über das Dritte Reich dieses mit der Metapher des für einen offenen oder latenten 
Toleranz und Demokratie

Bürgerkrieg stehenden Behemoth charakterisiert (Neumann 1977), den die etatistischen Konservativen vergeblich durch den Leviathan ersetzen wollten.

\section{Bewährungsproben des liberalen Pluralismus}

Dagegen hat das Nachfolgemodell des erfolgreichen Versuches, in den Niederlanden und in England Toleranz und Bürgerrechte nicht vom Staat, sondern von der Gesellschaft her zu etablieren, im 20. Jahrhundert eine viel erfolgreichere Entwicklung durchlaufen. Zwar erreichte diese einen Tiefpunkt, als in den 40er-Jahren nur wenige Länder in Europa wie Großbritannien, Schweden, Irland und die Schweiz ihre liberalen und demokratischen Errungenschaften behaupten konnten. Doch nach 1945 und dann in einem zweiten Schub nach 1989 setzte ein weltweiter Siegeszug der liberalen Demokratie und des ihm zugrunde liegenden pluralistischen Modells ein. Selbstverständlich spielten bei diesem Vorgang historisch kontingente Umstände eine entscheidende Rolle. Doch sollten dabei Strukturmerkmale des pluralistischen Modells nicht unbeachtet bleiben, die erheblich zur hegemonialen Akzeptanz der liberalen Demokratie beigetragen haben.

Schulemachend für Deutschland wurde in der Nachkriegszeit die Pluralismustheorie Ernst Fraenkels, die er in seinem Buch „Deutschland und die westlichen Demokratien“ (1968) entwickelte: Sie ist ohne den engen Konnex von Toleranz und Demokratie als Partizipationshandeln der Bürger nicht zu denken. Seit Fraenkels einflussreicher Neuformulierung des Pluralismus in der Frühphase der Bundesrepublik Deutschland ist es üblich geworden, in Rousseaus identitärer Demokratiekonzeption die analytische Kontrastfolie zum Pluralismus-Modell zu sehen (Fraenkel 1968: 173-180). Zunächst ist gegen eine solche Sichtweise einzuwenden, dass Rousseau zentrale Elemente seiner antipluralistischen Homogenitätskonzeption der politischen Theorie des Hobbes entlehnt hat: Diesem kommt also in der theoretischen Fundierung des Antipluralismus zeitliche Priorität gegenüber jenem zu. Vor allem aber eignet sich der Hobbes'sche Ansatz auch deswegen besser als Gegenbegriff zum Pluralismus, weil er wie dieser auf dem Repräsentationsgedanken ebenso aufbaut wie auf der Möglichkeit seiner Anwendung auf Großflächenstaaten.

In dem Maße, wie Rousseau jede Form der Stellvertretung der Aktivbürger ablehnte und die entscheidende Funktionsbedingung seiner Demokratie kleinflächige Territorien mit einer wenig differenzierten sozialen und kulturellen Ordnung waren, verbietet sich eigentlich deren Vergleich mit dem von Fraenkel konzipierten, auf moderne Massengesellschaften bezogenen Pluralismus. Geben wir aber aus analytischen Gründen dem etatistischen Ordnungsmodell der französischen Politiques und dem theoretisch ausdifferenzierten Muster des Hobbes'schen Leviathan mitsamt der von ihnen propagierten Toleranz-Konzeption sowie deren Aktualisierung im Sinne des Antipluralismus Carl Schmitts den Vorzug, so liegt eine zweite Modifikation des Fraenkel'schen Ansatzes nahe. Als dessen Vorläufer hatte dieser die Genossenschaftstheorie Otto von Gierkes (Fraenkel 1968: 170 f., 182 f.) einerseits und Harold Laskis frühen radikalpluralistischen Ansatz (Fraenkel 1968: 171 f.) andererseits genannt. Zwar ist diese Zuordnung nicht falsch, aber sie greift zu kurz. In langfristiger historischer Perspektive sind die gemeinsamen Schnittmengen von Fraenkels pluralismustheoretischem 
Ansatz mit dem liberalen Toleranzmuster viel evidenter, wie es sich im Aufstand der Niederlande und in der Englischen Revolution historisch konkretisierte.

Folgende Argumente sprechen für eine solche Analogisierung. Das in der Frühen Neuzeit dem Staat vorgelagerte Grundrecht auf Religions- und Meinungsfreiheit konstituierte das entscheidende Fundament der pluralistischen Arena des 20. Jahrhunderts, weil es eine Sphäre des Privaten begründete, in der auch die kollektiven Organisationen der Bürger, also die Parteien und Verbände, verankert sind. Erst aufgrund der aus der Privatheit resultierenden Toleranz konnten die religiösen Kräfte konsensual die Regeln festlegen, nach denen sie koexistieren wollten. In analoger Weise existiert im pluralistischen Modell zwar der Staat als juristischer Souverän weiter, indem er kraft seiner legislativen Kompetenz die Kompromisse der gesellschaftlichen Kräfte als Gesetze durchsetzt und die von ihnen gewollten Regeln kodifiziert, in deren Rahmen der pluralistische Interessenkonflikt stattfindet. Aber inhaltlich nimmt er keinen Einfluss auf die Interessenartikulation selbst, welche in die Kompetenz der autonom agierenden, aber grundsätzlich privaten Parteien und Verbände fällt (Fraenkel 1968: 166). Ferner funktionierte das Toleranz-Modell in den Niederlanden und in England nach dem Muster des Zusammenspiels von Heterogenität und Homogenität. Die Heterogenität bestand darin, dass auf horizontaler Ebene die spezifische Glaubensidentität gewahrt blieb. Doch deren Grenze resultierte aus der religiösen Identität der anderen Gruppierungen. Garant der Einhaltung dieser Grenze war im vertikalen Politikraum das juristische Monopol des Staates, der das Außenverhalten der religiösen Gruppen insofern homogenisierte, als die staatlich garantierten Spielregeln jede Privilegierung ausschlossen. Dieses Dualsystem aber gehört auch zum Herzstück des Fraenkel'schen Pluralismusmusters.

Tatsächlich unterschied Fraenkel zwischen einem kontroversen und einem nichtkontroversen Sektor innerhalb seines Pluralismus-Modells. Im kontroversen Bereich findet der Interessenkonflikt der organisierten Gruppen um die Verteilung des disponiblen Teils des Bruttosozialprodukts statt. Der aus ihm resultierende Kompromiss lässt sich in der geometrischen Figur des Parallelogramms der Kräfte abbilden, dessen Diagonale als Orientierungsnorm die Richtung des Kompromisses angibt, der entweder unterhalb der staatlichen Ebene in einen Tarifvertrag oder auf ihr in einem vom Parlament verabschiedeten Gesetz seine endgültige Gestalt erhält. Der nichtkontroverse Sektor dagegen ist definiert durch die von allen Beteiligten geteilten Spielregeln, Verfassungsnormen und den gemeinsamen in der politischen Kultur verankerten Werten, aber auch durch den Konsens ,über zahlreiche detaillierte Fragen der Wirtschaft, Gesellschaft und Politik“ (Fraenkel 1968: 168). Wie im idealtypischen Toleranzschema der autonomen religiösen Pluralität in der Frühen Neuzeit ist die Existenz eines nichtkontroversen Sektors die Garantie dafür, dass die heterogene Gesellschaft ihr Korrektiv in einem Minimum normativer Homogenität findet. Bricht dieser Basiskonsens zusammen, so ist ihre extremste Konsequenz der Bürgerkrieg, dessen Potenzialität auch in diesem Modell keineswegs verdrängt wird (Fraenkel 1968: 64, 168).

Es gehört zur Signatur des herrschenden Zeitgeistes, dass die Vertreter der sogenannten Postmoderne den Generalangriff auf jenes Minimum an Homogenität gemeinsamer Überzeugungen und Interessen eröffneten, denen Fraenkel mit seinem Konzept des nichtkontroversen Sektors ein institutionelles Profil verlieh. Was in normativer und auch in empirischer Hinsicht längst als der unverzichtbare Kitt ausgewiesen ist, ohne den die Gesellschaft in ihre 
Elemente zerfiele, wurde im Namen der Differenz als Abgleiten in den Totalitarismus stigmatisiert. Doch brachte der postmoderne Druck auf den Fraenkel'schen nichtkontroversen Sektor die Konsequenz einer innovativen Legitimation der Moderne hervor. Sowohl die Habermas'sche Idee, ,symmetriestiftende Normen könnten einem herrschaftsfreien Diskurs entspringen“ (Euchner 1991: 132), als auch Rawls „Theorie der Gerechtigkeit“ fungieren längst als philosophische Fundierung des nicht-kontroversen Sektors. Für dessen Absicherung sieht Jürgen Habermas ein Testverfahren der „Kommunikationsgemeinschaft der Betroffenen" (Habermas 1973: 144 f.) vor, an dessen Schluss die Generalisierbarkeit bzw. die Verallgemeinerung der Interessen und damit ihre „Richtigkeit“ steht. Und John Rawls grenznutzentheoretisches Modell gelangt zu diesem Ergebnis, wenn bei fortdauernder sozialer und wirtschaftlicher Ungleichheit diese zum Vorteil der schwächsten Glieder der Gesellschaft führt (Rawls 1975: 27 ff.).

Niemand kann diesen beiden Ansätzen ernsthaft totalitäre Engführungen unterstellen, zumal es ihr Ziel ist, gerade die Hindernisse der pluralen Entfaltung zu beseitigen. Allerdings folgen sie Fraenkel, wenn sie das Erreichen dieses Ziels abhängig machen von einer aposteriorischen Zusammenführung der partikularen Differenzen. Walter Euchner hat in diesem Zusammenhang darauf hingewiesen, in der Diskussion um die Postmoderne werde zumeist vergessen, ,daß die wichtigsten politischen Pluralismuskonzeptionen nicht dem postmodernen Diskurs entsprungen sind, sondern einem spezifischen Bemühen um politische Modernität, nämlich dem Versuch Harold Laskis, die Staatssouveränität zu depotenzieren, und Ernst Fraenkels Kampf um ein richtiges Verständnis der Strukturprinzipien einer, modernen Demokratie“ in Deutschland. Es war Fraenkel, der immer betont hat, Pluralismus könne nur gedeihen, wenn ideelle und institutionelle Voraussetzungen und Rahmenbedingungen weithin akzeptiert würden“" (Euchner 1991: 132 f.).

Euchners Hinweis auf die konsensuale Basis des neopluralistischen Ansatzes leitet abschließend über zu der Frage, in welchem Verhältnis er zur Dialektik von Relativismus und Fundamentalismus heute steht.

\section{Die Dialektik von Relativismus und Fundamentalismus}

$\mathrm{Zu}$ Recht ist gegen die bereits dargestellte Pluralismuskritik von rechts eingewandt worden, die organisierten Verbände hätten sich faktisch als Stabilisierungsfaktor der liberalen Demokratie erwiesen, indem sie den konstruktiven Dialog mit der Ministerialbürokratie bei der Formulierung von Gesetzesvorlagen und umgekehrt suchten. Wie Wilhelm Hennis einst prägnant formulierte, verstieße es gegen fundamentale Interessen der Verbände selbst, den Staat zu kolonisieren: Sie schlachteten dann die Kuh, von deren Milch sie lebten (Hennis 1961: 78). Doch schwerer fällt die Pluralismuskritik von links ins Gewicht. Sie zeigt die Grenzen des politischen Pluralismus unter kapitalistischen Bedingungen auf. Danach sind die organisierten Partikularinteressen privilegiert, die in einem Immediatverhältnis zu den Funktionsmechanismen der kapitalistischen Wirtschaft stehen, während die anderen Interessenlagen strukturell vernachlässigt und marginalisiert werden (Offe 1971: 155-189). Harold Laski zog daraus den Schluss, einen wirklich gerechten Pluralismus könne es daher nur unter den Bedingungen des demokratischen Sozialismus geben. Doch lässt sich gegen 
diese Kritik einwenden, dass Fraenkel für das Funktionieren des Pluralismus in bürgerlichen Gesellschaften ein erhebliches Maß an sozialer Gerechtigkeit einklagte: In der sozialstaatlichen Umverteilung von oben nach unten sah er eine entscheidende Funktionsvoraussetzung seines Modells. Aber liegt nicht gerade in dieser wirtschaftlichen und sozialen Stoßrichtung seine Antiquiertheit?

Gewiss war Fraenkel weit davon entfernt, dem Verhältnis von Politik und Religion ,,angesichts der Möglichkeit einer technischen Reproduktion des Menschen durch den Menschen und zu seinen Zwecken“ (Stein 2007: 145) eine solche zentrale Bedeutung beizumessen, wie es heute vielfach geschieht. ${ }^{1}$ Aber es spricht für die Robustheit seines neopluralistischen Modells, dass es auch auf die gegenwärtige Situation anwendbar erscheint. Denn ihm muss zugestanden werden, dass es im Schatten sich zuspitzender kultureller und religiöser Fragmentierungen durchaus in der Lage ist, tragfähige Antworten auf die Probleme des Relativismus und des Fundamentalismus zu geben, wie sich insbesondere am Beispiel der islamischen Herausforderung in den westlichen Metropolen zeigen lässt (Pfahl-Traugbehr 2007: 110-119). Es ist oft darauf hingewiesen worden, dass sowohl postmoderner Relativismus als auch religiöser Fundamentalismus Ausfluss der Moderne sind. Mit der Zerstörung der traditionalen Lebenswelten durch die Industrialisierung ging ein Prozess der Entwurzelung einher, der die Einzelnen auf sich selbst zurückwarf und ihnen die Freiheit gab, aber auch die Last zumutete, sich jenseits traditionaler Gewissheiten kraft der eigenen kritischen Vernunft selbst zu orientieren. Dieser Schritt in die individuelle Autonomie gelingt jedoch nicht allen und eröffnet dadurch dem Fundamentalismus eine Chance, die Einzelnen in ein System scheinbarer Glaubensgewissheiten einzubinden, deren Preis die bedingungslose Unterwerfung unter eine totalitäre Instanz ist (Berger: 2008: 5-16). In der unmittelbaren Nachkriegszeit musste es Fraenkel darauf ankommen, die autonome Organisation partikularer Interessen als Signum einer freien und offenen Gesellschaft angesichts der immer noch nachwirkenden obrigkeitsstaatlichen Traditionen in Deutschland zu legitimieren und durchzusetzen. Heute hat sich der sozio-politische Kontext verändert: Gefahr droht nicht primär aus der Reprimierung des Partikularen, sondern aus dessen Hegemonie, wie die Destruktion universaler Normen durch die Postmoderne und bedenkliche religiöse, kulturelle und soziale Polarisierungen sowie Ansätze zu Parallelgesellschaften zeigen. Wie ist sie zu bannen, ohne auf das gescheiterte Instrumentarium des etatistischen Antipluralismus zurückgreifen zu müssen?

Mit großer Weitsicht hat Fraenkel einem Pluralismuskonzept, das - wie die Postmoderne - nur den kontroversen Sektor zulässt, ebenso eine Absage erteilt wie dem Fundamentalismus, der die absolute Vorherrschaft der Homogenität notfalls mit totalitären Mitteln anstrebt. Sein Ziel ist demgegenüber die Vielfalt in der Einheit. Letztere wird dadurch erreicht, dass der konsensuale Wille der Gesellschaft selbst als Hüter des nichtkontroversen Sektors fungiert. Zu Recht hat das Bundesverfassungsgericht im bekannten Kopftuchstreit den Verbotsantrag der baden-württembergischen Landesregierung mit dem Argument zurückgewiesen, dass diese Entscheidung nicht von ihm, sondern dort zu regeln sei, wo sie hingehört, „nämlich in die Gesellschaft und in die Parlamente. Das Volk muss sich durch seine von ihm

1 Vgl. neben der Monografie von Stein 2007 u. a.; Audi/Wolterstorff 1997; Habermas 2001; Willems 2003; 2004; Habermas/Ratzinger 2005. 
gewählten Vertreter darüber verständigen, wohin die Reise gehen soll. Es muss nach weiterer Aufhellung der tatsächlichen Hintergründe und Abwägen des Für und Wider anhand politischer Maßstäbe darüber entscheiden, wie wir in Zukunft mit den Hervorbringungen, Riten und Zeichen umgehen wollen, die über drei Millionen bei uns lebender Menschen islamischen Glaubens als Teil ihrer Persönlichkeit und Würde mitgebracht haben. Das Kopftuch im öffentlichen Dienst ist insoweit nur die Spitze des Eisberges“ (Hassemer 2004: 28).

Auch die übergeordnete Norm, die dieser Suche als regulatives Prinzip des nichtkontroversen Sektors zu dienen hat, ist mit dem Artikel 1 des Grundgesetzes verfassungsmäßig kodifiziert: „Die Würde des Menschen ist unantastbar“, eine Norm, welche nicht nur auf den traditionellen Nationalstaat, sondern auch auf die im Entstehen begriffene Weltgesellschaft übertragen werden kann. Aus diesem Artikel des Grundgesetzes folgen nicht nur die Gebote, sondern auch die Grenzen, welche den Pluralismus „einhegen“ und ihn vor dem Abgleiten in den Relativismus der Indifferenz bewahren. Peter L. Berger hat sie auf einen Katalog praktischen Verhaltens im konkreten Lebensbezug heruntergebrochen, ohne den eine kulturellreligiöse Vielfalt im Spannungsfeld zwischen Relativismus und Fundamentalismus nicht möglich wäre: „die Fähigkeit, zwischen dem Glaubenskern und marginalen religiösen Inhalten, die kognitive Zugeständnisse zulassen, unterscheiden zu können; die Bereitschaft, sich mit der historisch-kritischen Analyse religiöser Schriften und Traditionen auseinanderzusetzen, durch die der Glaube übermittelt wurde; die Fähigkeit, die Berechtigung von Zweifeln an den Dogmen der eigenen Konfession einzuräumen; die Fähigkeit, Menschen, die nicht zur eigenen Glaubensgemeinschaft gehören, nicht als Feinde zu betrachten und bereit zu sein, mit diesen Menschen in friedlicher Koexistenz zu leben“ (Berger 2008: 10).

Es mag ja sein, dass diese universalisierbaren Normen bestimmten Varianten des Zeitgeistes widersprechen, in denen der Zweifel und der Relativismus und in seinem Gefolge als dialektische Antwort der Fundamentalismus in seiner religiösen und säkularen Spielart zu dominieren scheinen. Doch dem ist entgegenzuhalten, dass die Menschenrechtserklärung der Vereinten Nationen von 1948, welche für alle Völker und Nationen das gemeinsame Ideal postuliert, Menschenrechte und Grundfreiheiten zu verwirklichen, angesichts der homogenisierenden und nivellierenden Tendenzen der entstehenden Weltgesellschaft ihre Zukunft noch vor sich hat. Früher oder später, so die keineswegs unbegründete Hoffnung, wird auch der einseitig das Vereinzelte und Unterschiedliche betonende Zeitgeist und der auf ihn reagierende totalitäre Fundamentalismus diesem Trend nachgeben und ,sich dem Pathos der Menschenrechtserklärung anschließen müssen. Sie ist Ausdruck dieser Universalkultur“" (Tönnies 2008: 13).

\section{Literatur}

Audi, Robert/Wolterstorff, Nicholas, 1997: Religion in the Public Square. The Place of Religious Convictions in Political Debate, Lanham/London.

Bast, Jürgen, 1999: Totalitärer Pluralismus. Zu Franz L. Neumanns Analysen der politischen und rechtlichten Struktur der NS-Herrschaft, Tübingen.

Berger, Peter L., 2008: Gewissheit - religiöse, moralische, politische, in: Friedrich-EbertStiftung (Hrsg.), Policy Paper, Juni 2008, 5-16. 
Broszat, Martin, 1986: Der Staat Hitlers. Grundlegung und Entwicklung seiner inneren Verfassung, München (11. Aufl.).

Euchner, Walter, 1981: Vorwort, in: Saage 1981, 9-10.

Euchner, Walter, 1987: Die Staatsphilosophie des Thomas Hobbes, Hagen.

Euchner, Walter, 1991: Postmoderner Pluralismus oder pluralistische Moderne, in: perspektiven ds. 8, 131-141.

Forst, Rainer, 2003: Toleranz im Konflikt. Geschichte, Gehalt und Gegenwart eines umstrittenen Begriffs, Frankfurt a. M.

Forsthoff, Ernst, 1933: Der totale Staat, Hamburg.

Fraenkel, Ernst, 1968: Deutschland und die westlichen Demokratien, Stuttgart/Berlin/Mainz (4. unveränderte Aufl.).

Habermas, Jürgen, 1973: Legitimationsprobleme im Spätkapitalismus, Frankfurt a. M.

Habermas, Jürgen, 2001: Glauben und Wissen. Friedenpreis des deutschen Buchhandels, Frankfurt a. M.

Habermas, Jürgen/Ratzinger, Joseph, 2005: Dialektik der Säkularisierung. Über Vernunft und Religion, Freiburg u. a.

Hassemer, Winfried, 2004: Religiöse Toleranz im Rechtsstaat. Das Beispiel Islam, München.

Hennis, Wilhelm, 1961: Verfassungsordnung und Verbandseinfluss. Bemerkungen zu ihrem Zusammenhang im politischen System der Bundesrepublik Deutschland, in: PVS 2, 25-35.

Hobbes, Thomas, 1984: Leviathan oder Stoff, Form und Gewalt eines kirchlichen und bürgerlichen Staates. Hrsg. und eingel. v. Iring Fetscher, Übersetzung: Walter Euchner, Frankfurt a. M.

Neumann, Franz, 1977: Behemoth. Struktur und Praxis des Nationalsozialismus 1933-1944. Hrsg. und mit einem Nachwort „Franz Neumanns Behemoth und die heutige Faschismusdiskusssion“ von Gert Schäfer. Aus dem Amerikanischen übersetzt von Hedda Wagner und Gert Schäfer, Köln/Frankfurt a. M.

Offe, Claus, 1971: Politische Herrschaft und Klassenstrukturen. Zur Analyse spätkapitalistischer Gesellschaftssysteme, Frankfurt a. M. (3. unveränderte Aufl.).

Pfahl-Traugbehr, Armin, 2007: Toleranz braucht Grenzen, in: vorgänge 46, 110-119.

Rawls, John, 1975: Eine Theorie der Gerechtigkeit, Frankfurt a. M.

Saage, Richard, 1981: Herrschaft, Toleranz, Widerstand. Studien zur politischen Theorie der Niederländischen und der Englischen Revolution, Frankfurt a. M.

Saage, Richard, 1987: Der italienische und der deutsche Faschismus, in: ders., Arbeiterbewegung, Faschismus, Neokonservatismus, Frankfurt a. M, 121-159.

Schmitt, Carl, 1938: Der Leviathan in der Staatslehre des Thomas Hobbes. Sinn und Fehlschlag eines politischen Symbols, Hamburg.

Schmitt, Carl, 1985: Der Hüter der Verfassung, Berlin.

Schnur, Roman, 1962: Die französischen Juristen im konfessionellen Bürgerkrieg des modernen Staates, Berlin.

Stein, Tine, 2007: Himmlische Quellen und irdisches Recht. Religiöse Voraussetzungen des freiheitlichen Verfassungsstaates, Frankfurt a. M./New York.

Tönnies, Sibylle, 2008: Die Menschenrechte - nichts als schöne Worte?, in: Frankfurter Allgemeine Sonntagszeitung, 6. Juli 2008, Nr. 27, S. 13. 
Willems, Ulrich, 2002: Religion als Privatsache?, in: Michael Minkenberg/Ulrich Willems (Hrsg.), Politik und Religion, PVS-Sonderheft 33, Wiesbaden, 303-328.

Willems, Ulrich, 2004: Weltanschaulich neutraler Staat, christlich-abendländische Kultur.

Zur Struktur und Konsequenzen in der Bundesrepublik, in: Manfred Walther (Hrsg.), Religion und Politik. Zur Theorie und Praxis des theologisch-politischen Komplexes, Baden-Baden, 303-328.

Korrespondenzanschrift:

Prof. i. R. Dr. Richard Saage

Dohnenstieg 6

14195 Berlin

E-Mail: saage@gmx.net

\section{Zivilgesellschaft in Europa}



\section{European Civil Society on the Road to Success?}

Herausgegeben von Dr. Matthias Freise

2008, 260 S., brosch., 34,-€,

ISBN 978-3-8329-3522-1

(European Civil Society, Bd. 1)

Das Konzept der Zivilgesellschaft hat in den vergangenen Jahren verstärkt Eingang in die europäische Governance-Forschung gefunden. Dieser Band führt theoretische und empirische Arbeiten zum Regieren im europäischen Mehrebenensystem zusammen und leistet einen Beitrag zum besseren Verständnis zivilgesellschaftlicher Entwicklungslinien in Europa.

Bitte bestellen Sie im Buchhandel oder versandkostenfrei unter $>$ www.nomos-shop.de

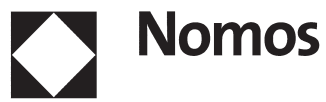

